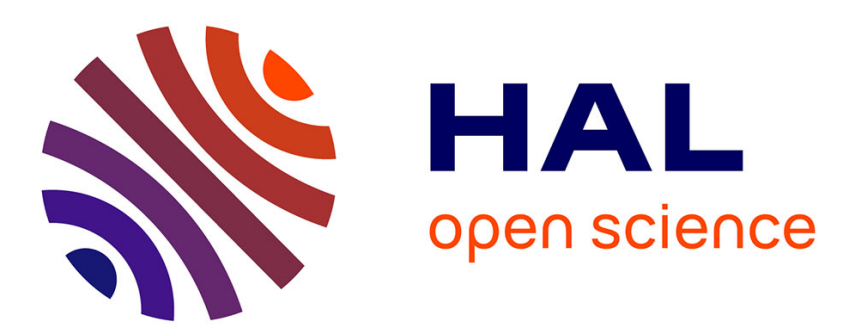

\title{
On discrete loop signatures and Markov loops topology Yves Le Jan
}

\section{To cite this version:}

Yves Le Jan. On discrete loop signatures and Markov loops topology. 2019. hal-02265642

\section{HAL Id: hal-02265642 \\ https://hal.science/hal-02265642}

Preprint submitted on 11 Aug 2019

HAL is a multi-disciplinary open access archive for the deposit and dissemination of scientific research documents, whether they are published or not. The documents may come from teaching and research institutions in France or abroad, or from public or private research centers.
L'archive ouverte pluridisciplinaire HAL, est destinée au dépôt et à la diffusion de documents scientifiques de niveau recherche, publiés ou non, émanant des établissements d'enseignement et de recherche français ou étrangers, des laboratoires publics ou privés. 


\title{
On discrete loop signatures and Markov loops topology
}

\author{
Yves Le Jan
}

August 11, 2019

\begin{abstract}
Our purpose is to explore, in the context of loop ensembles on finite graphs, the relations between combinatorial group theory, loops topology, loop measures, and signatures of discrete paths. We determine the distributions of the loop homotopy class, and of the first and second homologies, defined by the lower central series of the fundamental group. This last result has yet to be extended to higher order homologies.
\end{abstract}

\section{Geodesic loops and fundamental group for graphs}

We consider a finite connected graph $\mathcal{G}$, denoting by $X$ the set of vertices and by $E^{o}$ the set of oriented edges.

The extremities of an oriented edge $e$ are denoted $\left(e^{-}, e^{+}\right)$. The opposite oriented edge is denoted $-e$.

Recall that on graphs, geodesics paths are defined as non backtracking paths: $\left(x_{0}, x_{1}, \ldots, x_{n}\right)$ with $\left\{x_{i}, x_{i+1}\right\}$ in $E$ and $x_{i-1} \neq x_{i+1}$. Based loops at $x$ are paths from $x$ to $x$. We say they are tailless if their first edge and the opposite of their last edge differ. Loops are equivalence classes of based loops under the shift. Geodesic loops are defined as equivalence classes of non backtracking tailless based loops under the shift.

For each choice of a base vertex $x$, the fundamental group (Cf [13] ) $\Gamma_{x}$ is defined by geodesics from $x$ to $x$. The composition rule is concatenation

0

${ }^{0}$ AMS 2000 subject classification: . 
followed by erasure of backtracking subarcs. These groups are all isomorphic to the free group $\Gamma$ with $r=|E|-|X|+1$ generators, in a non canonical way. However, geodesic (unrooted) loops are in canonical bijection with the conjugacy classes of any $\Gamma_{x}$.

The isomorphisms between different $\Gamma_{x}$ 's, as well as a set of generators for the free group, can be defined by the choice of a spanning tree $T$ of the graph. To each oriented edge $\alpha=(a, b)$ outside the spanning tree we associate the element $\gamma_{x}(\alpha)$ of $\Gamma_{x}$ defined by the based loop obtained by concatenation of the geodesic from $x$ to $\alpha^{-}$in $T$, $\alpha$, and the geodesic from $\alpha^{+}$to $x$ in $T$. Note that $\gamma_{x}(-\alpha)$ is the inverse of $\gamma_{x}(\alpha)$. Finally recall that each loop $l$ is homotopic to a unique geodesic loop $l^{g}$, obtained by removing from the loop all its subtrees. The same holds for based loops (but in this case the tail is not removed).

\section{The lower central series}

Recall (see section 5-3 in [10], section 6-4 in [14]) that the lower central series $\Gamma^{(n)}$ of normal subgroups of the free group $\Gamma$ are defined recursively by setting $\Gamma^{(1)}=\Gamma$ and $\Gamma^{(n+1)}=\left[\Gamma^{(n)}, \Gamma\right]$. The quotients groups $H_{n}=\Gamma^{(n)} / \Gamma^{(n+1)}$ are Abelian. $H_{1}$ is the homology group of the graph, i.e the abelianized image of the fundamental group. By Witt's formula (Cf theorem 5-11 in [10]), $H_{n}$, we will refer to as the homology group of class $n$, is a free Abelian group with $d_{n}=\frac{1}{n} \sum_{d \mid n} \mu(d) r^{n / d}$ generators, $\mu$ denoting the Moebius function. In particular, $d_{1}=r, d_{2}=\frac{r(r-1)}{2}$, and $d_{3}=\frac{r^{3}-r}{3}$.

The quotient group $\Gamma / \Gamma^{(n+1)}$ is the free nilpotent group of class $n$, with $r$ generators (obtained by imposing that all iterated commutators of order $n+1$ vanish).

Each element $u$ of $\Gamma^{(n)}$ projects on an element $h_{n}(u)$ of $H_{n}$. which depends only on the conjugacy class of $u$, as $h_{n}\left(u_{1} u_{2}\right)=h_{n}\left(u_{1}\right)+h_{n}\left(u_{2}\right)$.

We can now define the degree $d(\gamma)$ of a geodesic loop $\gamma$ as the highest index $n$ such that the associated conjugacy class is included in $\Gamma^{(n)} . h(\gamma)$ denotes the corresponding element of $H_{d(\gamma)}$. Note that $h_{n}(\gamma)$ vanishes for $n<d(\gamma)$.

\section{Discrete signatures}

Given a root vertex $x_{0}$, a spanning tree $T$ and an orientation $e_{j}$ of the of the $r=|E|-|X|+1$ edges $\pm e_{j}$ not included in $T$, the elements $\gamma_{x_{0}}\left(e_{j}\right)$ denoted $\gamma_{j}$ generate the free group $\Gamma_{x_{0}}$. 
Each group element $g$ (except the neutral element) can be expressed uniquely by a reduced word $\gamma_{j_{1}}^{n_{1}} \gamma_{j_{2}}^{n_{2}} \ldots \gamma_{j_{l}}^{n_{m}}, m$ being a positive integer and the $n_{i}$ a $m$ tuple of non zero integers (reduced means that consecutive $j$ 's are distinct). Each conjugacy class, or equivalently geodesic loop, is represented uniquely by a class of cyclically reduced words equivalent under the shift (cyclically reduced means reduced, and that either $j_{1} \neq j_{m}$, either that $j_{1}=j_{m}$ and $\left.\operatorname{sgn}\left(n_{1}\right)=\operatorname{sgn}\left(n_{m}\right)\right)$.

Following the definition given in corollary 5-19 of [10], we will associate to such a goup element the formal series $S(g)=\prod_{i=1}^{l} e^{n_{j_{i}} X_{j_{i}}}$, the $X_{j}$ 's being non-commuting symbols.

By analogy with the definition of Chen [2], extended to bounded variation paths in [3], and in the theory of rough paths, we say that $S(g)$ is the signature of $g$. Note that the connection with this notion becomes more visible if one lifts the geodesic based loop to a geodesic (I.e. non backtracking) path of the universal Abelian cover, which is a lattice. Shift invariance becomes invariance under Verwaat transformation.

We denote by $\mathfrak{L}$ the free Lie algebra (Cf [10], section 5-6 or [14] Section 0-2 ) generated by the $X_{j}$ 's. It is the space of formal series whose homogeneous terms of all degrees are Lie polynomials.

Let us start by recalling a few fundamental properties proved in [10] and [14]) :

\section{Proposition 1}

a) $S\left(g_{1} g_{2}\right)=S\left(g_{1}\right) S\left(g_{2}\right)$

b) $\log (S(g))$ belongs to $\mathfrak{L}$.

c) The sum of the terms of lowest degree in $S(g)-1$ and in $\log (S(g))$, denoted $P_{g}(X)$ are equal, and their degree is $d(g) . P_{g}$ is a homogeneous Lie polynomial of degree $d(g)$.

d) For any word $u$ composed of the non-commuting symbols $X_{j}$, denoting by $\amalg$ the shuffle product of words (Cf [14], section 1-4), and $\langle S(g), u\rangle$ the coeficient of $u$ in $S(g)$,

$$
\left\langle S\left(g_{1}\right) S\left(g_{2}\right), u\right\rangle=\sum_{u_{1}, u_{2}, u_{1} \uplus u_{2}=u}\left\langle S\left(g_{1}\right) u_{1}\right\rangle\left\langle S\left(g_{2}\right), u_{2}\right\rangle .
$$

Remark 1 It follows easily from the above proposition that $P_{g^{-1}}(X)=-P_{g}(X)$ (as $S(g) S\left(g^{-1}\right)=1$ and that $P_{g}(X)$ and $d(g)$ depends only on the conjugacy class $C(g)$. 
If $\gamma$ is a geodesic loop, we denote by $P_{\gamma}$ the Lie polynomial defined by the associated conjugacy class. If $l$ is a loop, we denote by $P_{l}$ the polynomial $P_{l^{g}}$ and its degree $d\left(l^{g}\right)$ by $d(l)$. $h\left(l^{g}\right)$ will be denoted $h(l)$ and for $n \leqslant d(l)$, $h_{n}\left(l^{g}\right)$ will be denoted $h_{n}(l)$.

As in [7], we denote by $N_{e}(l), e \in E^{o}$, the oriented edge occupation field defined by a loop $l$ and by $N_{e}(\mathcal{L})=\sum_{l \in \mathcal{L}} N_{e}(l)$ the field defined by a set of loops $\mathcal{L}$. It counts the total number of traversals of $e$ and therefore verifies the Eulerian property: We set $\check{N}_{x, y}=N_{x, y}-N_{y, x}$. One checks easily from the Eulerian property that for any choice of spanning tree and outside edges orientation, $N$ is determined by the $r$ integers $\widetilde{N}_{e_{i}}$ we will simply denote by $\check{N}_{i}$.

Then we have:

\section{Proposition 2}

a) $d(l)>1$ iff $\check{N}_{i}(l)=0$ for all $1 \leqslant i \leqslant r$.

b) If $d(l)=1, P_{l}=\sum_{1 \leqslant i \leqslant r} \check{N}_{i}(l) X_{i}$.

c) If $d(l) \geqslant 1, h_{1}(l)=\sum_{1 \leqslant i \leqslant r} \check{N}_{i}(l) h_{1}\left(\gamma_{i}\right)$.

Proof. Note that $\breve{N}_{i}(l)=\breve{N}_{i}\left(l^{g}\right)$. Then compute the non constant term of degree 1 in $S\left(l^{g}\right)$ and $h_{1}(l)=h_{1}\left(l^{g}\right)$ using the decomposition of the signature in a product of exponentials stemming from the decomposition of $l^{g}$ by a cyclically reduced word.

For $l$. any based loop, we denote by $N_{e(1), e(2), \ldots, e(m)}(l)$ the number of increasing $m$-tuples of times at which $l$. crosses the $m$-tuple oriented edges $e(1) \ldots e(m)$ successively. Denote by $\breve{N}_{i, j}$ the number $N_{e_{i}, e_{j}}+N_{-e_{i},-e_{j}}-N_{e_{i},-e_{j}}-$ $N_{-e_{i}, e_{j}}$. Then:

\section{Proposition 3}

a) If $h_{1}(l)$ vanishes (i.e. if $d(l) \geqslant 2$ ), and if $l$. is any based loop representing the loop $l, \check{N}_{i, j}(l)-\breve{N}_{j, i}(l)$ is independent of the base point and $d(l)>2$ iff $\tilde{N}_{i, j}(l)-\breve{N}_{j, i}(l)=0$ for all $1 \leqslant i<j \leqslant r$.

b) If $d(l)=2, P_{l}=\frac{1}{2} \sum_{1 \leqslant i<j \leqslant r}\left(\check{N}_{i, j}(l)-\check{N}_{j, i}(l)\right)\left[X_{i}, X_{j}\right]$.

c) If $d(l) \geqslant 2, h_{2}(l)=\frac{1}{2} \sum_{1 \leqslant i<j \leqslant r}\left(\check{N}_{i, j}(l)-\check{N}_{j, i}(l)\right) h_{2}\left(\left[\gamma_{i}, \gamma_{j}\right]\right)$. 
Proof. Let $l$. be a loop based at $x_{0}$ representing $l$ and $l^{g}$ be the associated geodesic based loop, defining an element $\gamma$ of $\Gamma_{x_{0}}$. Using the decomposition of the signature in a product of exponentials, we get that the sum of the terms of degree $\leqslant 2$ in $S(\gamma)$ equals:

$$
1+\sum_{1 \leqslant i \leqslant r} \check{N}_{i}(l) X_{i}+\frac{1}{2} \sum_{1 \leqslant i \leqslant r} \frac{1}{2}\left(N_{i}(l)\left(N_{e_{i}}(l)-1\right)+N_{-e_{i}}(l)\left(N_{-e_{i}}(l)-1\right)-\right.
$$
$\left.2 N_{e_{i}}(l) N_{-e_{i}}(l)\right) X_{i}^{2}+\sum_{1 \leqslant i<j \leqslant r}\left(N_{i, j}(l) X_{i} X_{j}+\check{N}_{i, j}(l) X_{j} X_{i}\right)+\frac{1}{2} \sum_{1 \leqslant i \leqslant r}\left(N_{e_{i}}(l)+\right.$ $\left.N_{-e_{i}}(l)\right) X_{i}^{2}$ (the second and third sum come from the products of terms of degree one in two exponentials and the last sum from the terms of degree two in one exponential )

$$
=1+\sum_{1 \leqslant i \leqslant r} \check{N}_{i}(l) X_{i}+\frac{1}{2}\left[\sum_{1 \leqslant i \leqslant r} \check{N}_{i}(l) X_{i}\right]^{2}+\frac{1}{2} \sum_{1 \leqslant i<j \leqslant r}\left(\check{N}_{i, j}(l)\left[X_{i}, X_{j}\right]+\right.
$$
$\left.\check{N}_{j, i}(l)\left[X_{j}, X_{i}\right)\right]$, denoting $X_{i} X_{j}-X_{j} X_{i}$ by $\left[X_{i}, X_{j}\right]$. Note also that the terms of degree $\leqslant 2$ in $\log (S(\gamma))$ are $\sum_{1 \leqslant i \leqslant r} \check{N}_{i}(l) X_{i}+\frac{1}{2} \sum_{1 \leqslant i<j \leqslant r}\left(\check{N}_{i, j}(l)-\right.$ $\left.\left.\check{N}_{j, i}(l)\right)\right)\left[X_{i} X_{j}\right]$. a) and b) follow directly, by remark 1 . Then c) follows from theorem 5-12 in [10] and its corollary.

Remark: More generally, we can define

$$
\check{N}_{i(1), i(2), \ldots, i(m)}(l .)=\sum_{\epsilon_{k}= \pm, 1 \leqslant k \leqslant m} \prod_{k=1}^{m} \epsilon_{k} N_{\varepsilon_{1} e_{i(1)}, \varepsilon_{2} e_{i(2)}, \ldots, \varepsilon_{m} e_{i(m)}} .
$$

Then, if $d(l)=m$, it follows directly from its definition that:

$$
P_{l}=\sum_{i(1), i(2), \ldots, i(m)} \check{N}_{i(1), i(2), \ldots, i(m)}(l .) X_{i(1)} X_{i(2)} \ldots X_{i(m)}
$$

for any representative $l$. of $l$, Moreover from theorem 1-4 in[14] (or in theorem 5-17 in [10] ) this last expression can be rewritten as follows:

\section{Proposition 4}

$$
P_{l}=\frac{1}{m} \sum_{i(1), i(2), \ldots, i(m)} \check{N}_{i(1), i(2), \ldots, i(m)}(l .)\left[\left[\ldots\left[X_{i(1)}, X_{i(2)}\right] \ldots\right] X_{i(m)}\right] .
$$

Remark: Equivalently, $P_{l}$ equals:

$$
\frac{1}{m} \sum_{i(1)<i(2), i(3), \ldots, i(m)}\left(\check{N}_{i(1), i(2), \ldots, i(m)}(l .)-\check{N}_{i(2), i(1), i(3), \ldots, i(m)}(l .)\right)\left[\left[\ldots\left[X_{i(1)}, X_{i(2)}\right] \ldots\right] X_{i(m)}\right] .
$$

This gives a non self-contained proof of proposition 3. In general, this expression can be further modified to get a decomposition in any specific basis of 
the free Lie algebra (cf [14], chapter 4). Then it follows that its coefficients which are linear combinations of $\check{N}^{\prime}$ 's depend only on $l$, as $\check{N}_{i}$, and $\check{N}_{i, j}$ if $d(l)>1$.

For example, if $d(l)=3$, using Jacobi identity, we get that $\left[\left[X_{k}, X_{i}\right] X_{j}\right]=$ $-\left[\left[X_{i}, X_{j}\right] X_{k}\right]-\left[\left[X_{j}, X_{k}\right] X_{i}\right]$. Hence $P_{l}=\frac{1}{3} \sum_{i<j<k}\left(\left[\left(\check{N}_{i, j, k}-\widetilde{N}_{j, i, k}-\widetilde{N}_{k, i, j}+\right.\right.\right.$ $\left.\check{N}_{i, k, j}\right](l)\left[\left[X_{i}, X_{j}\right] X_{k}\right]+\left[\left(\check{N}_{j, k, i}-\check{N}_{k, j, i}-\check{N}_{k, i, j}+\check{N}_{i, k, j}\right](l)\left[\left[X_{j}, X_{k}\right] X_{i}\right]\right)+\frac{1}{3} \sum_{i \neq j}\left[\check{N}_{i, j, i}-\right.$ $\left.\check{N}]_{j, i, i}\right](l)\left[\left[X_{j}, X_{i}\right] X_{i}\right]$. Morevover, as $\check{N}_{j}(l)=0, \check{N}_{i, j, k}+\check{N}_{i, k, j}+\check{N}_{j, i, k}=$ $\check{N}_{j} \stackrel{N}{i, k}=0$ and as $\check{N}_{j}(l)=0, \check{N}_{j, k, i}+\check{N}_{k, i, j}+\check{N}_{k, j, i}=0$. Therefore:

$P_{l}=\frac{1}{3} \sum_{i<j<k}\left(\left[\left(\check{N}_{j, i, k}+2 \check{N}_{k, i, j}\right](l)\left[\left[X_{j}, X_{i}\right] X_{k}\right]+\left[\left(2 \check{N}_{j, k, i}+\check{N}_{i, k, j}\right](l)\left[\left[X_{j}, X_{k}\right] X_{i}\right]\right)+\right.\right.$ $\frac{2}{3} \sum_{i \neq j} \check{N}_{i, j, i}(l)\left[\left[X_{j}, X_{i}\right] X_{j}\right]$.

\section{Loop measures and homotopies distribu- tion.}

Following [7], we attach a positive conductance $C_{e}$ to each edge $e \in E$ and a killing rate $\kappa_{x}$ to each vertex $x \in X$, then, denoting $\Lambda$ a set of $r$ oriented edges define the duality measure $\lambda_{x}=\kappa_{x}+\sum_{y} C_{x, y}$

and the $\lambda$-symmetric transition matrix $P_{y}^{x}=\frac{C_{x, y}}{\lambda_{x}}, P_{\Delta}^{x}=\kappa_{x} \lambda_{x}$. The energy functional is:

$$
\epsilon(f, f)=\frac{1}{2} \sum_{x, y} C_{x, y}(f(x)-f(y))^{2}+\sum_{x} \kappa_{x} f(x)^{2}
$$

and the associated Green function is denoted by $G$.

We define a measure $\mu$ on (discrete time, unbased) loops:

$$
\mu(l)=\frac{1}{\operatorname{mult}(l)} \prod_{\text {edges of } l}\left(P_{e^{+}}^{e^{-}}\right)^{\operatorname{mult}(l)} .
$$

Here $\operatorname{mult}(l)$ denotes the multiplicity of the loop $l$. Note that

$$
|\mu|=\mu(1)=-\log (\operatorname{det}(I-P))
$$

Note that this measure is induced by the restriction to non-trivial discrete loops of the measure $\sum_{x \in X} \int_{0}^{\infty} \frac{1}{t} \mathbb{P}_{t}^{x, x} \lambda_{x} d t$ defined on continuous time based loops, $\mathbb{P}_{t}^{x, x}$ being the non-normalized bridge measure defined by the transition semigroup $\exp (t[I-P])$ associated with the energy functional (Cf $[7])$. It is the discrete space version of the loop measure defined by Lawler and 
Werner ([6] and Symanzik ([16]).

We denote by $\mathcal{L}_{\alpha}$ the Poisson point process of intensity $\alpha \mu$. The ensemble $\mathcal{L}_{\alpha}$ can be decomposed into independent sets of loops of distinct homotopies: For any geodesic loop $\gamma$, the number of loops $l \in \mathcal{L}_{\alpha}$ such that $l^{g}=\gamma$ is a Poisson variable of parameter $\mu_{\gamma}$.

In the case of the regular graphs with unit conductances and constant $\kappa$, a simple expression of $\mu_{\gamma}$ was obtained in [12]:

Proposition 5 If $\mathcal{G}$ is a d-regular graph, with $C_{e}=1, \kappa$ constant, for any closed geodesic $\gamma$, the number of loops homotopic to $\gamma$ is a Poisson r.v. of expectation:

$$
\mu_{\gamma}=\frac{1}{\operatorname{mult}(\gamma)}\left(\frac{d+\kappa}{2(d-1)}\left(1-\sqrt{\left.1-\frac{4(d-1)}{(d+\kappa)^{2}}\right)}\right)^{|\gamma|}\right.
$$

In particular, for $\kappa=0, \mu_{\gamma}=\frac{1}{\text { mult }(\gamma)}(d-1)^{-|\gamma|}$

Remark: If $\kappa=\frac{1}{u}+u(d-1)-d$, the associated generating function $\sum_{\gamma} u^{|\gamma|} \mu_{\gamma}$ coincides with the logarithm of Ihara's zeta function (Cf [15], [4] [7]).

$$
\sum_{\gamma} \frac{1}{\operatorname{mult}(\gamma)} u^{|\gamma|}=\log \left(\zeta_{I h}(u)\right)=-\log \left[\left(1-u^{2}\right)^{-\chi} \operatorname{det}\left(I-u A+u^{2}(d-1) I\right)\right]
$$

where $A$ denotes the adjacency matrix and $\chi$ the Euler number $|E|-|X|$ of the graph.

This result follow from a more general one: If $(x, y)$ is an edge, let us denote $r^{x, y}$ the probability that the Markov chain starting at $y$ returns to $y$ following a tree-contour subloop without visiting $x$ at the first step. Note that:

$$
r^{x, y}=\sum_{z \neq x} P_{z}^{y} P_{y}^{z} \sum_{n=0}^{\infty}\left[r^{y, z}\right]^{n}
$$

Set $\rho^{x, y}=\sum_{n=0}^{\infty}\left[r^{x, y}\right]^{n}$. We get the following:

Proposition 6 If $\gamma$ varies in the set of geodesic loops (conjugacy classes), $\left|\left\{l \in \mathcal{L}, l^{g}=\gamma\right\}\right|$ are independent Poisson r.v. with mean values

$$
\mu_{\gamma}=\frac{1}{\operatorname{mult}(\gamma)}\left(\prod_{\text {edges of } \gamma} P_{e^{+}}^{e-} \rho^{e^{-}, e^{+}}\right)^{m u l t(\gamma)}
$$


and

$$
\rho^{x, y}=1+\sum_{z \neq x} P_{z}^{y} \rho^{y, z} P_{y}^{z} \rho^{x, y}
$$

If $\mathcal{G}$ is a $d$-regular graph, with $C_{e}=1, \kappa$ constant, it is clear that the $r^{x, y}$ are all equal to the same return probability to the root of a half- $d$-regular tree. We then get from the previous equations that

$$
\rho^{x, y}=\frac{(d+\kappa)^{2}}{2 s(d-1)}\left(1-\sqrt{1-\frac{4(d-1)}{(d+\kappa)^{2}}}\right)
$$

and recover the result of [12].

This argument is close to the proof of Ihara's formula in [15].

The corresponding generalization of Ihara's formula is given in [1]. A different generalization was given in [17].

Let us now consider the distribution of the number of loops homotopic to a point; It is obviously a Poisson distribution of parameter $-\ln (\operatorname{det}(I-$ $P)-\sum_{\gamma} \mu_{\gamma}$.

To compute this quantity, let us now denote $r^{x, y, k}$ the probability that the Markov chain starting at $y$ returns to $y$ for the first time in $2 k$ steps following a tree-contour subloop without visiting $x$ at the first step. Set $r^{x, y}(s)=$ $\sum r^{x, y, k} s^{k}$. Set $\rho^{x, y}(s)=\sum_{n=0}^{\infty}\left[r^{x, y}(s)\right]^{n}$

Note that:

$$
r^{x, y}(s)=s \sum_{z \neq x} P_{z}^{y} P_{y}^{z} \rho^{y, z}(s)
$$

and that $\rho^{x, y}(s)$ satisfies the relation:

$$
\rho^{x, y}(s)=1+s \sum_{z \neq x} P_{z}^{y} \rho^{y, z}(s) P_{y}^{z} \rho^{x, y}(s) .
$$

Let us now denote $r^{x, k}$ the probability that the Markov chain starting at $x$ returns to $x$ for the first time in $2 k$ steps following a tree-contour subloop. Set $r^{x}(s)=\sum r^{x, k} s^{k}$ Note that:

$$
r^{x}(s)=s \sum_{y} P_{y}^{x} P_{x}^{y} \rho^{x, y}(s) .
$$

Let denote $\rho^{x, k}$ the probability that the Markov chain starting at $x$ returns to $x$ in $2 k$ steps following a tree-contour subloop. Set $\rho^{x}(s)=\sum_{0}^{\infty} \rho^{x, k} s^{k}$ and note that: $\rho^{x}(s)=\frac{1}{1-r^{x}(s)}$. 
The number of loops of $\mathcal{L}$ homotopic to a point is a Poisson r.v. with expectation $\sum_{x} \sum_{0}^{\infty} \frac{1}{2 k} \rho^{x, k} s^{k}=\sum_{x} \int_{0}^{1} \frac{\rho^{x}(s)-1}{2 s} d s$.

If $\mathcal{G}$ is a $d$-regular graph, with $C_{e}=1, \kappa$ constant, it is clear that $\rho^{x, y}(s)$ and $\rho^{x}(s)$ are constants in the edge or vertex variables. We get from the previous equations that

$$
\begin{gathered}
\rho^{x, y}(s)=\frac{(d+\kappa)^{2}}{2 s(d-1)}\left(1-\sqrt{1-\frac{4 s(d-1)}{(d+\kappa)^{2}}}\right) \text { and } \\
\rho^{x}(s)=\frac{2(d-1)}{\left.d-2+d \sqrt{1-\frac{4 s(d-1)}{(d+\kappa)^{2}}}\right)} .
\end{gathered}
$$

From the expression of $\rho^{x}$, by an elementary integration, we finally deduce that:

Proposition 7 If $\mathcal{G}$ is a d-regular graph, with $C_{e}=1, \kappa$ constant, the number of loops homotopic to a point is a Poisson r.v. of expectation

$$
|X|\left(\frac{d}{2}(\log (2)-\log (b+1))+(d-2)\left(\log \left(b+\frac{d-2}{d}\right)-\log \left(1+\frac{d-2}{d}\right)\right)\right)
$$

with $b=\sqrt{1-4 \frac{d-1}{(d+\kappa)^{2}}}$.

In particular, for $\kappa=0$, this is equal to: $(d-2) \log (d-2)+d / 2) \log (d)-$ $(d-2+d / 2) \log (d-1)$.

\section{$5 \quad$ Homology and holonomies distributions}

The loops $\mathcal{L}_{\alpha}$ can also be classified into independent sets of loops $\mathcal{L}_{\alpha}^{(d)}$ according to their degrees $d\left(l^{g}\right)$. For each degree $d$, we can try to determine the distribution of the sum of the homologies of the loops of degree $d$ and the distribution of the number of loops of given $d$-th homology,

In this section, we recall and complete the results obtained in [7], [9], and [11], which solve the problem for $d=1$.

Define $\eta_{x, y}^{j}=1_{x=e_{j}^{-}, y=e_{j}^{+}}-1_{y=e_{j}^{-}, x=e_{j}^{+}}$.

Denoting by $P^{(\theta)}$ the matrix $P_{y}^{x} e^{2 \pi \sqrt{-1} \sum \theta_{i} \eta_{x, y}^{i}}$, we have (see [7]):

$$
\int\left(e^{\sum_{i} \sqrt{-1} \pi \breve{N}_{i}(l) \theta_{i}}-1\right) \mu(d l)=-\log \left(\operatorname{det}\left(I-P^{(\theta)}\right) .\right.
$$

Hence for any $\left(j_{i}\right) \in \mathbb{Z}^{r}$, using an inverse Fourier transform, we have: 
Proposition $8\left|\left\{l \in \mathcal{L}_{\alpha}, \check{N}_{i}(l)=j_{i}, i=1 \ldots r\right\}\right|$ are independent Poisson r.v. with expectations:

$$
\alpha \mu\left(\left\{l, \check{N}_{i}(l)=j_{i}, i=1 \ldots r\right\}\right)=-\alpha \int_{[0,1]^{r}} \log \left(\operatorname{det}\left(I-P^{(\theta)}\right)\right) \prod_{i=1}^{r} e^{-2 \pi \sqrt{-1} j_{i} \theta_{i}} d \theta_{i} .
$$

Remarks:

- Consequently, the distribution of the homology field defined by $\mathcal{L}_{\alpha}$ is:

$$
\left.\left.P\left(\check{N}_{i}\left(\mathcal{L}_{\alpha}\right)=j_{i}, i=1 \ldots r\right)=\int_{[0,1]^{r}}\left[\frac{\operatorname{det}(I-P)}{\operatorname{det}\left(I-P^{(\theta)}\right)}\right]\right)\right]^{\alpha} \prod_{i=1}^{r} e^{-2 \pi \sqrt{-1}, j_{i} \theta_{i}} d \theta_{i}
$$

- An intrinsic, but less explicit, expression ( not relying on the choice of the spanning tree) is given in [9]. It involves the evaluation of a discrete differential form ( $\mathrm{Cf}[7]$, section 1-5) on the loop. The Fourier integration is done on the Jacobian torus ([5]), i.e. the quotient of the space of harmonic one-forms $H^{1}(\mathcal{G}, \mathbb{R})$ (i.e. the space of one-forms $\omega$ such that $\sum_{y} C_{x, y} \omega^{x, y}=0$ ) for all $x \in X$ by $H^{1}(\mathcal{G}, \mathbb{Z})$ the space of harmonic one-forms with $\mathbb{Z}$-valued integrals on loops. The Lebegue measure is normalized by its volume which is equal to $\sqrt{\operatorname{det}(J)}$, with $J_{i, j}=\delta_{i, j} C_{e_{i}}-C_{e_{i}} K_{e_{i}, e_{j}} C_{e_{j}}$, for $1 \leqslant i, j \leqslant r, K$ denoting the transfer matrix: $K_{e, f}=G_{e^{+}, f^{+}}+G_{e^{-}, f^{-}}-G_{e^{+}, f^{-}}-G_{e^{-}, f^{+}}$.

- For $\alpha=1$, an alternative expression (without inverse Fourier transform) is given in section 3 of [8].

To try to to solve the problem for higher values of $d$, in particular for $d=2$, we need to recall more results.

For the fundamental groups $\Gamma_{x}$ morphisms in a group $G$ are obtained from maps $A$, assigning to each oriented edge $e$ an element $A[e]$ in $G$ with $A[-e]=A[e]^{-1}$.

A path, in particular a based loop, is mapped to the product of the images by $A$ of its oriented edges and the associated loop $l$ to the conjugacy class of this product, i.e. the holonomy of $l$, is denoted $H_{A}(l)$. Moreover $H_{A}(l)=H_{A}\left(l^{g}\right)$

A gauge equivalence relation between assignment maps is defined as follows: $A_{1} \sim A_{2}$ iff there exists $Q: X \mapsto G$ such that:

$$
A_{2}[e]=Q\left(e^{+}\right) A_{1}[e] Q^{-1}\left(e^{-}\right)
$$

Equivalence classes are $G$-connexions. They define $G$ - Galois coverings of $\mathcal{G}$ (cf [11]). Obviously, holonomies depend only on connections. 
Given a spanning tree $T$, there exists a unique $A^{T} \sim A$ such that $A^{T}[e]=I$ for every edge $e$ of $T$.

For any unitary representation $\pi$ of $G$, denote $\chi_{\pi}(C)$ the normalized trace of the image by $\pi$ of any element in the conjugacy class $C$.

Recall that free groups are conjugacy separable: Two conjugacy classes are separated by a morphism in some finite group.

Conjugate separability implies that if we consider all unitary representations of finite groups and all connections, the holonomies determine the geodesic loop (i.e. the conjugacy class of $\Gamma$ ) defined by $l$. The functions $\gamma \mapsto \chi_{\pi}\left(H_{A}(\gamma)\right)$ span an algebra and separate geodesic loops.

Fix now a finite group $G$, and let $\mathcal{R}$ denote the set of irreducible unitary representations of $G$.

Define an extended transition matrix $P^{A, \pi}$ with indices in $X \times\{1,2, \ldots \operatorname{dim}(\pi)\}$ by $\left[P^{A, \pi}\right]_{y, j}^{x, i}=P_{y}^{x}[\pi(A[(x, y)])]_{j}^{i}$. Then the following proposition follows directly from the expression of the based loop measure inducing $\mu$ (see [7]):

\section{Proposition 9}

$$
\sum_{l} \chi_{\pi}\left(H_{A}(l)\right) \mu(l)=-\frac{1}{\operatorname{dim}(\pi)} \log \left(\operatorname{det}\left(I-P^{A, \pi}\right)\right)
$$

Remarks:

- This result extends to compact groups.

- For any unitary representation $\pi$, choose, for any oriented edge $e_{j}$, an Hermitian matrix $H_{j}^{(\pi)}$, such that $\exp \left[\sqrt{-1} H_{j}^{(\pi)}\right]=\pi\left[A\left(e_{j}\right)\right]$. Then, for any based loop representative of $l$, denoted $l$., the holonomy can be expressed as the normalized trace of the signature series acting on the matrices $H_{j}^{(\pi)}$ in place of the $X_{j}$ 's:

$$
\chi_{\pi}\left(H_{A}(l)\right)=\frac{1}{\operatorname{dim}(\pi)} \operatorname{Tr}\left(S\left(l_{.}^{g}\right)\left[H_{j}, 1 \leqslant j \leqslant r\right] .\right.
$$

- It follows from this proposition and group representation theory (Cf for example [18]) that $\left|\left\{l \in \mathcal{L}_{\alpha}, H_{A}(l)=C\right\}\right|$ are independent Poisson r.v. with expectations:

$$
\alpha \mu\left(\left\{l, H_{A}(l)=C\right\}\right)=-\alpha \sum_{\pi \in \mathcal{R}} \overline{\chi_{\pi}(C)} \frac{|C|}{|G|} \operatorname{dim}(\pi) \log \left(\operatorname{det}\left(I-P^{A, \pi}\right)\right) .
$$




\section{Nilpotent holonomy and homology of class two}

Let us now consider the case where $G$ is the free nilpotent group of class two based on the field $\mathcal{Z}_{p}=\mathcal{Z} / p \mathcal{Z}$, for some prime number $p$. This group can be defined as follows:

$$
\begin{aligned}
& G=\left\{(a, c), a \in \mathbb{Z}_{p}^{r}, c \in\left[\mathbb{Z}_{p}^{r}\right]^{\wedge 2}\right\}, \text { with product : } \\
& (a, c) \cdot\left(a^{\prime}, c^{\prime}\right)=\left(a+a^{\prime}, c+c^{\prime}+\left(a \otimes a^{\prime}-a^{\prime} \otimes a\right)\right) .
\end{aligned}
$$

Associativity is checked easily. The neutral element is $(0,0)$, and $(a, c)^{-1}=$ $(-a,-c)$.

For any $(r, r)$ skew-symetric matrix $h_{i, j}$ with coeficients in $\mathbb{Z}_{p}$, a unitary representation $U_{h}$ of $G$ on the space $V_{r, p}$ of functions on $\mathbb{Z}_{p}^{r}$ is defined as follows:

$$
U_{h}(a, c) \psi(x)=e^{\frac{2 \pi \sqrt{-1}}{p}(\langle c, h\rangle+\langle a, x\rangle)} \psi(x+h \cdot a)
$$

with $\langle c, h\rangle=\sum_{1 \leqslant i<j \leqslant r} h_{i, j} c_{i, j},(h \cdot a)_{i}=\sum_{1 \leqslant j \leqslant r} h_{i, j} a_{j}$ and $\langle a, x\rangle=\sum_{1 \leqslant i \leqslant r} a_{i} x_{i}$ ( note that $\left\langle a \otimes a^{\prime}-a^{\prime} \otimes a, h\right\rangle=\left\langle a^{\prime}, h \cdot a\right\rangle$ ). This is similar to the Schrödinger representation of the Heisenberg group.

$\operatorname{dim}\left(V_{r, p}\right)=p^{r}$ and an orthonormal base of $V_{r, p}$ is given by products of exponentials $\psi_{n_{1}, \ldots, n_{r}}\left(l_{1}, \ldots l_{r}\right)=e^{\frac{2 \pi \sqrt{-1}}{p}} \sum_{1 \leqslant i \leqslant r} l_{i} n_{i}$, with $0 \leqslant l_{i}<p$. We can check that the normalized trace $\chi_{U_{h}}((a, c))=1_{\{a=0\}} e^{\frac{2 \pi \sqrt{-1}}{p}\langle c, h\rangle}$.

Consider the $G$-connection $A$ defined by assigning to each edge $e_{i}, i \in$ $\{1, \ldots r\}$ the element $\left(v_{i}, 0\right), v_{i}$ being the $i$-th element of the canonical base of $\mathbb{R}^{r}$.

Then if $l$. is any based loop in $l,\left(\check{N}_{i}(l), \check{N}_{i, j}(l)-.\check{N}_{j, i}(l).\right)$ is a representative of $H_{A}(l)$ in $G$ and $\chi_{U_{h}}\left(H_{A}(l)\right)=1_{\left\{l, \check{N}_{i}(l)=0, \forall i\right\}} e^{\frac{2 \pi \sqrt{-1}}{p} \sum_{1 \leqslant i<j \leqslant r} \check{N}_{i, j}(l) h_{i, j}}$. By the previous proposition, $\sum_{l} \chi_{U_{h}}\left(H_{A}(l) \mu(l)=-\frac{1}{p^{r}} \log \left(\operatorname{det}\left(I-P^{A, U_{h}}\right)\right)\right.$. Hence, for any $(r, r)$ skew-symetric matrix $u_{i, j}$ with coeficients in $[0,1)$, $\sum_{\left\{l, \check{N}_{i}(l)=0, \forall i\right\}} e^{2 \pi \sqrt{-1} \sum_{1 \leqslant i<j \leqslant r} \check{N}_{e_{i}, e_{j}}(l) u_{i, j}} \mu(l)=\sum_{\left\{l, h_{1}(l)=0\right\}} e^{2 \pi \sqrt{-1}\left\langle h_{2}(l), u\right\rangle} \mu(l)$ $=\lim _{p \uparrow \infty}-\frac{1}{p^{r}} \log \left(\operatorname{det}\left(I-P^{A, U_{h(u, p)}}\right)\right.$, taking for $h(u, p)_{i, j}$ the integral part of $u_{i, j} p$.

Proposition $10\left|\left\{l \in \mathcal{L}_{\alpha}^{(1)}, \check{N}_{i, j}(l)=m_{i, j}, 1 \leqslant i<j \leqslant r\right\}\right|$ are independent 
Poisson r.v. with expectations:

$\alpha \mu\left(\left\{l, \check{N}_{i, j}(l)=m_{i, j}, 1 \leqslant i<j \leqslant r\right\}\right)=-\alpha \int_{[0,1] \frac{r(r-1)}{2}} F(u) \prod_{i, j} e^{-2 \pi \sqrt{-1} m_{i, j} u_{i, j}} d u_{i, j}$.

with $F(u)=\lim _{p \uparrow \infty} \frac{1}{p^{r}} \log \left(\operatorname{det}\left(I-P^{(A, h(u, p))}\right)\right)$.

Remarks:

- The inverse Fourier transfom can also be performed before taking the limit $p \uparrow \infty$

- As before, a more intrinsic formulation can be given using a couple of harmonic discrete differential forms and an integration on the product of two Jacobian tori. - The distribution of the homology field of class $2 h_{2}\left(\mathcal{L}_{\alpha}^{(1)}\right)$ can then be derived straightforwardly in the same way as the distribution of $h_{1}\left(\mathcal{L}_{\alpha}\right)$.

- The distributions of higher order homologies are likely to be obtained in a similar way, using representations of nilpotent groups of higher class.

\section{References}

[1] Nalini Anantharaman. Some relations between the spectra of simple and non-backtracking random walks. arXiv:1703.03852.

[2] Kuo-tsai Chen, Integration of paths-a faithful representation of paths by noncommutative formal power, Trans. Amer. Math. Soc. 156, 395-407 (1971).

[3] Ben Hambly, Terry Lyons, Uniqueness for the signature of a path of bounded variation and the reduced path group, Ann. of Math. (2), 171 109-167 (2010).

[4] Motoko Kotani, Toshikazu Sunada. Zeta functions of finite graphs. J. Math. Sci. Univ. Tokyo 7, 7-25 (2000).

[5] Motoko Kotani, Toshikazu Sunada. Jacobian Tori Associated with a Finite Graph and Its Abelian Covering Graphs. Advances in Applied Mathematics 24, 89-110. (2000).

[6] Gregory Lawler, Wendelin Werner. The Brownian loop soup. Probability Th. Rel. Fields 128, 565-588 (2004). 
[7] Yves Le Jan. Markov paths, loops and fields. École d'Été de Probabilités de Saint-Flour XXXVIII - 2008. Lecture Notes in Mathematics 2026. Springer. (2011).

[8] Yves Le Jan. Random flows defined by Markov loops. To appear in Stéminaire de Probabilités.

[9] Yves Le Jan. Markov loops, free field and Eulerian networks. arXiv:1405.2879. J. Math. Soc. Japan 67, 1671-1680 (2015).

[10] Wilhelm Magnus, Abraham Karass, Donald Solitar. Dover. (1976) Combinatorial group theory. 2d Ed. Dover. (1976).

[11] Yves Le Jan. Markov loops, Coverings and Fields. arXiv: 1602.02708. Annales de la Faculté des Sciences de Toulouse XXVI, 401-416 (2017).

[12] Pavel Mnëv. Discrete Path Integral Approach to the Selberg Trace Formula for Regular Graphs. Comm. Math. Phys. 274, 233-241 (2007).

[13] William S. Massey, Algebraic Topology: An Introduction Springer (1967).

[14] Christophe Reutenauer. Free Lie algebras. Clarendon Press. Oxford. (1993).

[15] Harold M. Stark, Audrey A. Terras, Zeta functions on finite graphs and coverings. Advances in Maths 121, 134-165 (1996).

[16] Kurt Symanzik, Euclidean quantum field theory. Scuola intenazionale di Fisica "Enrico Fermi". XLV Corso. 152-223 Academic Press. (1969);

[17] Yusuke Watanabe and Kenji Fukumizu, Graph zeta function in the Bethe free energy and loopy belief propagation. Adv. Neural Inform. Proc. Sys. 22, 2017-2025.(2010).

[18] Don Zagier. Appendix in Graphs on Surfaces and their Applications, by S.K. Lando and A.K. Zvonkin. Springer. (2004).

NYU-ECNU Institute of Mathematical Sciences at NYU Shanghai.

Département de Mathématique. Université Paris-Sud. Orsay

yves.lejan@math.u-psud.fr yl57@nyu.edu 